\title{
Aspirin-sensitive asthma and rhinosinusiti
}

\author{
Shuaib Nasser
}

angestion of aspirin or an NSAID induces heproducible reaction in sensitive patients wit asthma. The reaction characteristically occurs withi 30 to 120 minutes. In any individual the form of th feaction is consistent and any combination $o$ kymptoms may occur including systemic upset wit ,facial flushing, perspiration and intense lethargy dhinorrhoea, nasal congestion, conjunctivitis an respiratory symptoms of cough and bronchospasm Some patients experience gastrointestinal symptom with vomiting, diarrhoea and abdominal discomfort In more sensitive individuals, or if a large dose ha been taken, a severe reaction may ensue with th development of respiratory arrest and shock. Despite the association between aspirin-sensitivity an asthma the relationship is not causal and indeed th patient may have never taken aspirin or NSAIDs Therefore, for patients with this profile, the ter taspirin-sensitive asthma (ASA) or aspirin-intoleran bsthma is preferred to aspirin-induced asthma, whic should be reserved for acute reactions precipitated $b$ gspirin ingestion and not the chronic day-to-da syndrome experienced by these patients. This is severe subtype of asthma and often very difficult $t$ treat.

,Typically, patients complain of severe asthma frequently requiring maintenance therapy with ora borticosteroids. They usually have rhinosinusitis wit prominent nasal congestion, anosmia and loss of tast resulting from intractable nasal polyposis. The nasa symptoms may be resistant to therapy and requir dreatment with corticosteroids together with repeate surgical removal of nasal polyps. Avoidance o NSAIDs does not have a beneficial or detrimenta effect on the course of the chronic asthma o shinosinusitis. The relationship between symptom and aspirin-sensitivity is often not appreciated by th gatient or physician thereby potentially restrictin treatment options

The diagnosis of ASA should be suspected in an patient with a characteristic symptom profile even i there is no history of an aspirin/NSAID-induce neaction. Skin prick tests to aspirin or NSAIDs $i$ these patients are of no value as IgE plays no role $i$ this condition. There are no other $\dot{\boldsymbol{m}}$ vitr tests fo yspirin-sensitivity and therefore aspirin challenge ma be required to confirm or exclude the diagnosis $i$ there is any doubt from the history. The preferre nhallenge route is to use inhaled lysine-aspirin as a alternative to oral aspirin and thereby provoke either Łocalized nasal or a bronchial reaction. With lysin aspirin the symptoms experienced are limited to th girways and therefore easily reversed $b$ bronchodilators as opposed to the systemic symptom elicited during oral aspirin challenge. The challeng fomprises of the administration of doubling doses o dysine-aspirin at thirty-minute intervals using a han held nebulizer linked to a breath-actuated dosimeter The challenge is continued until there is a decrease $\mathrm{i}$ FE ${ }_{1}$ bf $20 \%$ compared to baseline. Nasa provocation can be used in patients suspected o aspirin-intolerant rhinitis or where poor lung functio precludes bronchial challenge.

ft is unusual for ASA patients to develo dermatological manifestations and aspirin induce articaria or angioedema should be considered different disorder

All NSAIDs, which cross-react with aspirin to caus entolerant reactions in ASA have anti-cyclooxygenas (COX) activity and the potency of a drug to inhibi COX $\dot{m}$ vitr eorrelates well with its ability to induc intolerant reactions ${ }^{1}$ Paracetamol (acetaminophen) i y weak inhibitor of cyclooxygenase and is generall yell tolerated but at doses of between 150 and $600 \mathrm{~m}$ induces symptoms in approximately $6 \%$ of aspirin Rensitive subjects. Our own experience and that fro recent published trials have demonstrated tha selective cyclooxygenase-2 (COX-2) inhibitors suc as rofecoxib and celecoxib are likely to be safe in th gnajority of patients with ASA strongly implicatin enhibition of the COX-1 isoenzyme of cyclooxygenas as the initiating event for ASA reactions ${ }^{2}$ Subject Evith ASA have urinary leukotriene (LT) ${ }_{4}$ tevels tha are several-fold higher than in normals or in aspirin tolerant asthmatics. However, there is substantia Gverlap of LT ${ }_{4}$ devels between these groups an Fherefore urinary LT ${ }_{4}$ eannot be used to diagnos aspirin sensitivity in an individual with asthma ${ }^{3}$ Apon challenge with aspirin, patients with AS Exhibit a further 4-fold increase in urinary LT ${ }_{4}$ Excretion accompanied by a decrease in FE ${ }_{1}$. Pretreatment with an inhibitor of leukotriene synthesi aot only inhibits this increase in cysteinyl leukotrien selease but also blocks the symptoms and sign resulting from aspirin ingestion ${ }^{4}$ The cysteiny dleukotrienes are potent bronchoconstrictors, an stimulate bronchial mucus secretion, increas yenopermeability and increase airwa ๓hyperresponsiveness in asthmatic individuals and ar therefore capable of producing all the symptoms an digns experienced by these subjects. Eosinophils an mast cells are found in greater numbers in th bronchial mucosa of ASA patient ${ }^{5}$ and eosinophils i ehese patients have a greater capacity to generat tcysteinyl leukotrienes compared to aspirin-toleran fisthmatics because there is increased expression o CT ${ }_{4}$ synthase, the terminal enzyme for the synthesi of cysteinyl leukotrienes ${ }^{6}$ Therefore these cell type are likely to be the source for the enhanced cysteiny leukotriene release in ASA

\section{Shuaib Nasse \\ Consultant physician}

Correspondence to

Dr S Nasse

Department of Allerg

Alinic 2

Addenbrooke's Hospital, Hills Road, Cambridge,

Prim Care Respir

$2002) 11(1$ ग15-1 
dherefore, inhibition of the iso-enzyme COX-1 is th s"affecter" or initiating event and cysteinyl leukotriene hre the "effectors" for the pathophysiologica grocesses leading to the symptoms and signs followin aspirin ingestion and for a significant proportion of th ghronic symptoms in ASA. However the connectin steps between COX-1 inhibition and activation of th 5-lipoxygenase pathway to generate cysteiny seukotrienes have not been fully elucidated. It ha been suggested that in ASA there is a relativ leficiency of the bronchodilator prostaglandin PGE In the respiratory tract compared to PGF2a, whic increases bronchial smooth muscle tone. Although there is no direct evidence for a relative deficiency o PGE2, inhaled PGE2 has been shown to completel drotect patients with ASA to a challenge with inhale yysine-aspirin and to prevent the increase in urinar ET $4^{7}$ The simplest hypothesis for the pathogenesi of ASA maintains that because both enzymes hav arachidonic acid as their substrate, inhibition of COX-1 leads to a diversion from the cyclooxygenas to the 5-lipoxygenase pathway. Although attractive there are a number of valid objections to this concep as it does not explain why inhibition of COX-1 doe tnot precipitate bronchoconstriction in aspirin-toleran asthmatics and does not explain the severe chroni symptoms experienced by patients with ASA dHowever, a combination of these hypotheses may lea eo a plausible theory. For example, a relativ fleficiency of PGE2 could result in upregulation o $\mathbb{C T}{ }_{4}$ synthase and therefore explain the chroni symptoms due to elevated basal cysteinyl leukotrien levels. Any additional decrease in PGE2 by COXinhibition would then divert arachidonic acid to the 5 hipoxygenase pathway leading to a further increase i eysteinyl leukotriene synthesis and to the acut Aymptoms experienced by subjects with AS following aspirin ingestion

Patients with ASA and rhinosinusitis should b warned to avoid the large number of drugs with -COX-1 inhibitory activity. Selective cyclooxygenase 2 (COX-2) inhibitors should be considered if treatmen is required for inflammatory disorders but should onl be introduced with caution until there is wide experience of these drugs in ASA. Aspiri flesensitization in ASA is an effective method o eontrolling symptoms of rhinosinusitis and th cegrowth of nasal polyps and may reduce systemi norticosteroid requirements with the possibility of a improvement in asthma control 8 Patients considere for desensitization are usually on large doses o sntranasal and inhaled corticosteroids for rhinosinusiti and asthma and are likely to require frequent course sof oral corticosteroids. Typically, desensitization take from 2 to 4 days and the patient continues on a dail fnaintenance dose of $600 \mathrm{mg}$. The state $o$

lesensitization is made possible because after eac gose of aspirin there is a refractory period durin which time a further equivalent dose produces $n$ dymptoms. The mechanism of this refractory perio gnd hence aspirin desensitization may be explained $b$ a combination of a downregulation of leukotrien airway receptor sensitivity during acute desensitizatio and reduced leukotriene production in response $t$ aspirin administration with chronic therapy ${ }^{9} \mathrm{dh}$ yefractory period is individual to each patient and ma kast from less than 2 days to more than 7 days and it $i$ gherefore essential to continue taking aspirin $600 \mathrm{~m}$ daily in order to prevent resensitisation. Aspiri blesensitization is a resource-intensive procedure wit attendant risks and should only be carried out in Hospital setting. Therefore, patients must be selecte yarefully and warned to discontinue aspirin if the develop gastro-intestinal or other adverse effects.

Although one would envisage that salicylate-free diet dould be useful in ASA, these have prove disappointing in ASA, but it is unclear whether this i due to poor compliance or the lack of accurate dietar advice. In addition, foods may also contain non acetylated salicylates, which do not inhibi cyclooxygenase and $\boldsymbol{i} n$ vitr dtudies have demonstrate that some of these may actually prevent aspirin fro acetylating cyclooxygenase thereby potentiall blocking aspirin-induced reactions. The patient' hsthma should be treated in the usual way wit bronchodilators and inhaled corticosteroids. Anti teukotrienes are a vital component in the managemen of patients with ASA. The two major classes of anti feukotrienes act either by blocking the effect o cysteinyl leukotrienes at the CysLT1 receptor (e.g zafirlukast, montelukast and pranlukast) or $\mathrm{b}$ preventing their biosynthesis by inhibition of th enzyme 5-lipoxygenase (e.g. zileuton - not available i the U.K.). A number of studies have established thei dfficacy in inhibiting aspirin-induce bronchoconstriction confirming the key role played $b$ systeinyl leukotrienes in the acute reaction. There $\mathrm{i}$ also evidence for clinical improvement with mor prolonged use of antileukotrienes resulting in a sncrease in respiratory function, fewer nasal symptom and a reduction in the number of asthm exacerbations ${ }^{\mathbb{1}}$

Despite optimal treatment, a significant proportion o patients with ASA will still require regular ora oorticosteroids for control of symptoms compared $t$ patients with other forms of asthma. The mos sroublesome nasal symptom is congestion and thi sisually requires treatment with topical corticosteroid and intermittent topical decongestants and may requir occasional courses of oral corticosteroids. Th addition of oral or nasal antihistamines may provid hdditional relief. Surgery for the removal of nasa polyps may be required on a regular basis and som patients may require polypectomies every few years Intractable cases should be referred to a specialis hllergy clinic or a respiratory physician wit experience of this condition.

\section{Reference}

1. Szczeklik A, Gryglewski RJ, Czerniawska Mysik G $\mathbb{R}$ elationship of inhibition of prostaglandi biosynthesis by analgesics to asthma attacks in aspiri sensitive patients. $\boldsymbol{B r}$ Med 1975 1:67-9.

В. Stevenson S. Lack of cross reactivity betwee hofecoxib and aspirin in aspirin sensitive patients wit asthma. J Allergy Clin Immunol 200180 :47-51. 
3. Smith CM, Hawksworth RJ, Thien FC, Christie PE Eee TH. Urinary leukotriene ${ }_{4}$ in bronchial asthma Eur Respir 1992 5:693-9.

4. Nasser SM, Bell GS, Foster S, bt a 5Effect of the dipoxygenase inhibitor ZD2138 on aspirin induce asthma. Thorax;1994:749-56.

5. Nasser SM, Pfister R, Christie PE, et al

Inflammatory cell populations in bronchial biopsie from aspirin sensitive asthmatic subjects. Am J Respi Crit Care Med:1996 35 :90-6.

6. Cowburn A, Sladek K, Soja J, et al Đverexpressio of leukotriene ${ }_{4}$ Bynthase in bronchial biopsies fro patients with aspirin intolerant asthma. 8 Clin Inves 199810 :834-46.

7. Sestini P, Armetti L, Gambaro G, et al dnhale IPGE2 prevents aspirin induced bronchoconstrictio
End urinary LT ${ }_{4}$ excretion in aspirin sensitive asthma Am J Respir Crit Care Med $1996 \mathbf{3 5}$ :572-5. 8. Sweet JM, Stevenson DD, Simon RA, Mathiso DA. Long term effects of aspirin desensitizatio treatment for aspirin sensitive rhinosinusitis asthma. $J$ Allergy Clin Immunol ;990 8 :59-65.

$\theta$. Nasser SM, Patel M, Bell GS, Lee TH. The effec Ef aspirin desensitization on urinary leukotriene 4 concentrations in aspirin sensitive asthma. Am đRespir Crit Care Me ;1995 15 :1326-30.

10. Dahlen, Nizankowska, Szczeklik, bt a s Benefit from adding the 5 lipoxygenase inhibitor zileuton $\mathrm{t}$ conventional therapy in aspirin intolerant asthmatics Am J Respir Crit Care Med 199815 :1187-94.

\section{Following on from the very successful first international primar care respiratory conference hosted by the GPIAG in Cambridg 2000 the IPCRG are delighted to announce the 2nd internationa primary care respiratory congres}

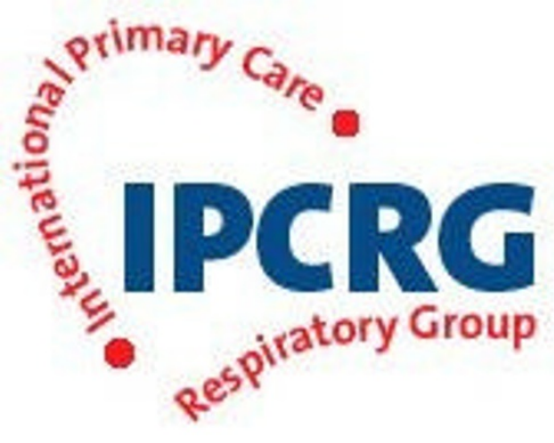

Congress, h 9 h June 2002, Amsterdam, The Netherland

isit the IPCRG website for further information

$$
\text { http://www.IPCRG.or }
$$

For registration information contact the congress office a

Telephone: + 3120525479

Bacsimile: + 3120525479 